\title{
Finite Groups Whose Maximal Subgroups Have the Hall Property
}

\author{
N. V. Maslova ${ }^{1 *}$ and D. O. Revin ${ }^{2 * *}$ \\ ${ }^{1}$ Institute of Mathematics and Mechanics UB RAS, Ekaterinburg, 620219 Russia; \\ Ural Federal University, Ekaterinburg, 620002 Russia \\ ${ }^{2}$ Sobolev Institute of Mathematics and Novosibirsk State University, Novosibirsk, 630090, Russia \\ Received March 3, 2012
}

\begin{abstract}
We study the structure of finite groups whose maximal subgroups have the Hall property. We prove that such a group $G$ has at most one non-Abelian composition factor, the solvable radical $S(G)$ admits a Sylow series, the action of $G$ on sections of this series is irreducible, the series is invariant with respect to this action, and the quotient group $G / S(G)$ is either trivial or isomorphic to $\mathrm{PSL}_{2}(7), \mathrm{PSL}_{2}(11)$, or $\mathrm{PSL}_{5}(2)$. As a corollary, we show that every maximal subgroup of $G$ is complemented.
\end{abstract}

DOI: $10.3103 / \mathrm{S} 105513441303005 \mathrm{X}$

Keywords: finite group, unsolvable group, maximal subgroup, Hall subgroup, complemented subgroup, normal series, Frattini subgroup, locally finite group, variety of groups.

\section{INTRODUCTION}

Throughout the article, except for Section 3, by a group we mean a finite group.

Recall that $H$ is a Hall subgroup of $G$ if the order of $H$ and the index of $H$ in $G$ are relatively prime.

We say that $G$ is a group whose maximal subgroups have the Hall property if every maximal subgroup of $G$ is a Hall subgroup. Let $\mathscr{V}$ denote the class of such groups.

A subgroup $H$ of a group $G$ is said to be complemented in $G$ if there exists its complement in $G$, i.e., a subgroup $K$ such that $H \cap K=1$ and $H K=G$.

We say that $G$ is a group with complemented maximal subgroups if every maximal subgroup of $G$ is complemented in $G$. Let $\mathscr{W}$ denote the class of such groups.

The study of groups in $\mathscr{V}$ and $\mathscr{W}$ was initiated by Levchuk and Likharev [16] and Tyutyanov [21]. It was proven that each non-Abelian simple group in $\mathscr{W}$ is isomorphic to either $\operatorname{PSL}_{2}(7) \cong \operatorname{PSL}_{3}(2)$, or $\mathrm{PSL}_{2}(11)$, or $\mathrm{PSL}_{5}(2)$. These are groups whose maximal subgroups have the Hall property, i.e., all these groups belong to $\mathscr{V}$. Tikhonenko and Tyutyanov [22] proved that each non-Abelian simple group in $\mathscr{V}$ is isomorphic to either $\mathrm{PSL}_{2}(7)$, or $\mathrm{PSL}_{2}(11)$, or $\mathrm{PSL}_{5}(2)$. They also conjectured that $\mathscr{V} \subseteq \mathscr{W}$. The above results show that each non-Abelian simple group in $\mathscr{V}$ belongs to $\mathscr{W}$. A similar assertion holds for solvable groups. Indeed, by [6, Ch. A, Proposition (10.5)]), the index of each maximal subgroup of a solvable group is primary; hence, every maximal subgroup of a solvable group is complemented by a Sylow subgroup. In [19], Monakhov studied finite solvable groups in $\mathscr{V}$ in more detail. He proved the following assertion.

Proposition 1 [19, Corollary 1]. For every finite solvable group $G$, the following conditions are equivalent.

(1) Each maximal subgroup of $G$ is a Hall subgroup.

(2) Each maximal subgroup of $G$ is complemented by a Sylow subgroup.

\footnotetext{
${ }^{*}$ E-mail: butterson@mail.ru

** E-mail: revin@math.nsc.ru
} 
(3) Each principal quotient of $G$ is isomorphic to a suitable Sylow subgroup of $G$.

He also formulated the following problem [19]: Describe non-Abelian composition factors of a finite unsolvable group whose maximal subgroups have the Hall property ${ }^{1}$. Later this problem was included in the Kourovka Notebook [18, Question 17.92]. In [17], the first author proved the following assertion: If a composition factor of a group in $\mathscr{V}$ is a non-Abelian simple group then it is isomorphic to either $\mathrm{PSL}_{2}(7)$, or $\mathrm{PSL}_{2}(11)$, or $\mathrm{PSL}_{5}(2)$. Hence, the above mentioned three groups exhaust (up to isomorphism) the class of non-Abelian composition factors of unsolvable groups in $\mathscr{V}$. This completely solves Monakhov's problem.

The next natural step is to obtain a complete description of groups in $\mathscr{V}$ and to verify the conjecture of Tikhonenko and Tyutyanov on the inclusion $\mathscr{V} \subseteq \mathscr{W}$. The present article is devoted to these questions.

We try to generalize Proposition 1 to arbitrary groups in $\mathscr{V}$. We reformulate this proposition as follows: For a solvable group $G$, we have $G \in \mathscr{V}$ if and only if $G$ admits a normal series

$$
G=G_{0}>G_{1}>\cdots>G_{n}=1
$$

such that $G_{i-1} / G_{i}$ is an elementary Abelian $p_{i}$-group for a suitable $p_{i} \in \pi(G)$, this quotient group is isomorphic to a Sylow $p_{i}$-group, and the action of $G / G_{i}$ on $G_{i-1} / G_{i}$ (regarded as a vector space) is irreducible. In particular, all Sylow subgroups of such a group $G$ are elementary Abelian groups and the Frattini subgroup is trivial.

A similar assertion need not hold for an unsolvable group in $\mathscr{V}$. Indeed, let $\mathscr{U}$ denote the class of finite groups $G$ satisfying the following conditions:

( $\mathscr{U} 1$ ) the Frattini subgroup $\Phi(G)$ of $G$ is a 3-group;

$(\mathscr{U} 2) \quad G / \Phi(G) \cong \operatorname{PSL}_{2}(7)$.

The following assertion shows the difference between the class of elementary Abelian groups and the class of Sylow subgroups of groups in $\mathscr{V}$.

Proposition 2. The following assertions hold.

(1) We have $\mathscr{U} \subseteq \mathscr{V}$, i.e., if $G \in \mathscr{U}$ then each maximal subgroup of $G$ is a Hall subgroup.

(2) We have $\mathscr{U} \subseteq \mathscr{W}$, i.e., if $G \in \mathscr{U}$ then each maximal subgroup of $G$ is complemented.

(3) If the Frattini subgroups of groups $G_{1}, G_{2} \in \mathscr{U}$ are non-trivial then $G_{1} / \Phi\left(\Phi\left(G_{1}\right)\right) \cong$ $G_{2} / \Phi\left(\Phi\left(G_{2}\right)\right)$.

(4) If $G \in \mathscr{U}$ and $\Phi(G) \neq 1$ then the elementary Abelian 3-group $\Phi(G) / \Phi(\Phi(G))$ is an irreducible 7 -dimensional $\mathbb{F}_{3}(G / \Phi(G))$-module with respect to the natural action of $G / \Phi(G) \cong \mathrm{PSL}_{2}(7)$. In particular, the least number of generators of $\Phi(G)$ is 7 .

(5) If a variety $\mathscr{B}$ consists of locally finite 3 -groups then there exists $G \in \mathscr{U}$ such that $\Phi(G)$ is a free group of rank 7 in $\mathscr{B}$.

(6) There exists $G \in \mathscr{U}$ such that each 7-generated 3-group is a homomorphic image of $\Phi(G)$.

(7) For every natural $n$, there exists $G \in \mathscr{U}$ such that the exponent of $\Phi(G)$ is greater than $n$

(8) For every natural $n$, there exists $G \in \mathscr{U}$ such that the degree of solvability of $\Phi(G)$ is greater than $n$.

In [24, Theorem 2, Corollary; 25, Theorem 2], Zel'manov suggests a positive solution of the restricted Burnside problem for groups of primary period. According to his results, for every natural $m$, the class of locally finite groups of period $3^{m}$ forms a variety of locally finite 3 -groups. By Proposition 2 , the universal 7 -generated finite group $B_{0}\left(7,3^{m}\right)$ of exponent $3^{m}$, which is free in this variety, is isomorphic to the Frattini subgroup of a suitable group in $\mathscr{U} \subseteq \mathscr{V}$.

The following assertion describes the class $\mathscr{V}$ and shows that the groups in $\mathscr{U}$ can be judged as exceptions. This is the main result of the present article.

\footnotetext{
${ }^{1}$ Although all simple groups in $\mathscr{V}$ are known and are composition factors of themselves, composition factors of an arbitrary group in $\mathscr{V}$ need not belong to $\mathscr{V}$.
} 
Theorem 1. A finite group $G$ is a group whose maximal subgroups have the Hall property if and only if $G$ admits a normal series ${ }^{2}$

$$
G=G_{0}>G_{1}>\cdots>G_{n}=1
$$

such that the following conditions hold.

(1) For $i>1$, the group $V_{i}=G_{i-1} / G_{i}$ is an elementary Abelian group that is isomorphic to a suitable Sylow subgroup of $G$ (in particular, each $G_{i}, i=0,1, \ldots, n$, is a Hall subgroup) and the action of $G / G_{i}$ by conjugations on $V_{i}$ (regarded as a vector space) is irreducible.

(2) For the quotient group $\bar{G}=G_{0} / G_{1}=G / G_{1}$, one of the following conditions holds:

(a) the order of $\bar{G}$ is prime;

(b) $\bar{G} \simeq \operatorname{PSL}_{5}(2)$;

(c) $\bar{G} \simeq \mathrm{PSL}_{2}(11)$;

(d) $\bar{G} \in \mathscr{U}$, i.e., $\Phi(\bar{G})$ is a 3-group and $\bar{G} / \Phi(\bar{G}) \simeq \operatorname{PSL}_{2}(7)$.

Corollary 1. Let $G$ be a finite group whose maximal subgroups have the Hall property. Then some Sylow tower of the solvable radical $S(G)$ is invariant with respect to the conjugation by elements of $G$ and the quotient group $G / S(G)$ is either trivial or isomorphic to $\mathrm{PSL}_{2}(7), \mathrm{PSL}_{2}(11)$, or $\mathrm{PSL}_{5}(2)$.

Theorem 1 allows us to confirm the conjecture of Tikhonenko and Tyutyanov.

Theorem 2. We have $\mathscr{V} \subseteq \mathscr{W}$. In other words, if $G$ is a group whose maximal subgroups have the Hall property then each maximal subgroup of $G$ is complemented.

The converse fails even for solvable groups ${ }^{3}$. Consider a finite group of prime exponent $p$ (for example, an elementary Abelian $p$-group). Each maximal subgroup is complemented but need not be a Hall subgroup. Examples of unsolvable groups possessing this property are $\mathrm{PGL}_{2}(7)$ and the direct product of $\mathrm{PSL}_{2}(7)$ and the cyclic group of order 3 . It is interesting to study groups in $\mathscr{W}$ in more detail. In particular, the following problem seems to be interesting.

Problem. Describe non-Abelian composition factors of groups with complemented maximal subgroups.

For all known examples of groups in $\mathscr{W}$, each non-Abelian composition factor is isomorphic to either $\mathrm{PSL}_{2}(7)$, or $\mathrm{PSL}_{2}(11)$, or $\mathrm{PSL}_{5}(2)$.

\section{DEFINITIONS, NOTATION, AND AUXILIARY RESULTS}

We use the standard notation and definitions that can be found in $[1,6,13,14,20]$.

Describing the structure of a finite group, we use the notation from [3].

For every set $\pi$ of prime numbers, let $\pi^{\prime}$ denote the set of prime numbers that do not belong to $\pi$. For every natural $n$, let $\pi(n)$ denote the set of prime divisors of $n$. For every finite group $G$, let $\pi(G)$ denote the set $\pi(|G|)$.

For every natural $n$ and prime $p$, by the $p$-part $n_{p}$ of $n$ we mean the greatest non-negative power of $p$ dividing $n$.

Let $\operatorname{Syl}_{p}(G)$ denote the set of Sylow $p$-subgroups of a group $G$.

We say that $H$ is a $\pi$-Hall subgroup of a group $G$, where $\pi$ is a set of prime numbers, if $\pi(H) \subseteq \pi$ and $\pi(|G: H|) \subseteq \pi^{\prime}$. Let $\operatorname{Hall}_{\pi}(G)$ denote the set of $\pi$-Hall subgroups of a group $G$.

\footnotetext{
${ }^{2}$ Although each $G_{i-1} / G_{i}$ is a principal quotient for $i>1$, the series need not be principal because the quotient group $G_{0} / G_{1}$ need not be simple.

${ }^{3}$ By Proposition 1, a finite solvable group $G$ is a group whose maximal subgroups have the Hall property if and only if each maximal subgroup of $G$ is complemented by a Sylow subgroup. As is mentioned above, each maximal subgroup of each simple group in $\mathscr{V}$ is complemented but this complement need not be a Sylow subgroup. Consider the group $\mathrm{PSL}_{2}(11)$. The normalizer of a Sylow 11 -subgroup (of order 55) and the normalizer of a Sylow 3 -subgrgoup (of order 12) are maximal subgroups and are complements of each other (see the table below).
} 
Let $G$ be a finite group. Then $S(G)$ denotes the solvable radical, $\Phi(G)$ denotes the Frattini subgroup (the intersection of all maximal subgroups), and $O_{\pi}(G)$ denotes the greatest normal $\pi$-subgroup, where $\pi$ is a set of prime numbers.

Let $\mathbb{F}_{q}$ denote the $q$-element field.

We use the following notation for groups:

$\operatorname{PSL}_{n}(q)$ is the projective special linear group of degree $n$ over the field $\mathbb{F}_{q}$;

$S_{n}$ is the symmetric group of degree $n$ and $A_{n}$ is the alternating group of degree $n$;

$D_{2 n}$ is the dihedral group of order $2 n$.

Lemma 1 [11, Lemma 1]. If $H$ is a $\pi$-Hall subgroup and $A$ is a normal subgroup of a group $G$ then $H A / A$ is a $\pi$-Hall subgroup of $G / A$ and $H \cap A$ is a $\pi$-Hall subgroup of $A$.

Lemma 2 [19, Lemma 4]. The classes $\mathscr{V}$ and $\mathscr{W}$ are closed under homomorphic images.

Proof. For $\mathscr{W}$, it suffices to observe that the preimage of a maximal subgroup is a maximal subgroup too. For $\mathscr{V}$, we use Lemma 1 together with the above observation.

Lemma 3 [16, Theorem 1, Lemmas 4 and 6; 21; 22]. For every finite simple group L, the following conditions are equivalent.

(1) We have $L \in \mathscr{V}$, i.e., each maximal subgroup of $L$ is a Hall subgroup.

(2) We have $L \in \mathscr{W}$, i.e., each maximal subgroup of $L$ is complemented.

(3) The group L is isomorphic to either $\mathrm{PSL}_{2}(7)$, or $\mathrm{PSL}_{2}(11)$, or $\mathrm{PSL}_{5}(2)$.

Lemma 4 [17, Theorem 1]. If $G$ is a group whose maximal subgroups have the Hall property then each non-Abelian composition factor of $G$ is isomorphic to either $\mathrm{PSL}_{2}(7)$, or $\mathrm{PSL}_{2}(11)$, or $\mathrm{PSL}_{5}(2)$.

For the proofs of the following two lemmas, the reader is referred to [3].

Lemma 5. Let $L \in\left\{\mathrm{PSL}_{2}(7), \mathrm{PSL}_{2}(11), \mathrm{PSL}_{5}(2)\right\}$. Then $|\mathrm{Out}(L)|=2$. In particular, we have $\pi(L)=\pi(\operatorname{Aut}(L))$.

Lemma 6. Let $L \in\left\{\mathrm{PSL}_{2}(7), \mathrm{PSL}_{2}(11), \mathrm{PSL}_{5}(2)\right\}$ and let $M$ be a maximal subgroup of $L$. The information on the structure, the order, and the index of $M$ is presented in the table below.

Table

\begin{tabular}{|c|c||c|c|c|}
\hline$L$ & $|L|$ & Structure of $M$ & $|M|$ & $|L: M|$ \\
\hline \hline $\mathrm{PSL}_{2}(7)$ & $2^{3} \cdot 3 \cdot 7$ & $S_{4}$ & $2^{3} \cdot 3$ & 7 \\
\hline & & $7: 3$ & $3 \cdot 7$ & $2^{3}$ \\
\hline \hline $\mathrm{PSL}_{2}(11)$ & $2^{2} \cdot 3 \cdot 5 \cdot 11$ & $A_{5}$ & $2^{2} \cdot 3 \cdot 5$ & 11 \\
\hline & & $11: 5$ & $5 \cdot 11$ & $2^{2} \cdot 3$ \\
\hline & & $D_{12}$ & $2^{2} \cdot 3$ & $5 \cdot 11$ \\
\hline \hline $\mathrm{PSL}_{5}(2)$ & $2^{10} \cdot 3^{2} \cdot 5 \cdot 7 \cdot 31$ & $2^{4}: \mathrm{PSL}_{4}(2)$ & $2^{10} \cdot 3^{2} \cdot 5 \cdot 7$ & 31 \\
\hline & & $2^{6}:\left(S_{3} \times \mathrm{PSL}_{3}(2)\right)$ & $2^{10} \cdot 3^{2} \cdot 7$ & $5 \cdot 31$ \\
\hline & & $31: 5$ & $5 \cdot 31$ & $2^{10} \cdot 3^{2} \cdot 7$ \\
\hline
\end{tabular}

Maximal subgroups of the groups $\mathrm{PSL}_{2}(7), \mathrm{PSL}_{2}(11)$, and $\mathrm{PSL}_{5}(2)$

Lemma 7. Let $L \in\left\{\mathrm{PSL}_{2}(7), \mathrm{PSL}_{2}(11), \mathrm{PSL}_{5}(2)\right\}$. If $p \in \pi(L)$ and $p$ divides the index of no maximal subgroup of $L$ then $(L, p)=\left(\mathrm{PSL}_{2}(7), 3\right)$. 
Proof. The indices of maximal subgroups of $\mathrm{PSL}_{2}(7), \mathrm{PSL}_{2}(11)$, and $\mathrm{PSL}_{5}(2)$ are presented in the table above.

Lemma 8. Assume that $L$ is a normal subgroup of a group $G$. Let

$$
L=L_{1} \times \cdots \times L_{n}
$$

be the direct power of either $\mathrm{PSL}_{2}(7)$, or $\mathrm{PSL}_{2}(11)$, or $\mathrm{PSL}_{5}(2)$. Put

$$
m= \begin{cases}2^{3} & \text { if } L_{i} \cong \mathrm{PSL}_{2}(7), \\ 2^{2} \cdot 3 & \text { if } L_{i} \cong \operatorname{PSL}_{2}(11), \\ 2^{10} \cdot 3^{2} \cdot 7 & \text { if } L_{i} \cong \operatorname{PSL}_{5}(2) .\end{cases}
$$

Then there exists a maximal subgroup $M$ of $G$ such that the index of $M$ divides $m^{n}$ and $G=L M$. In particular, if $L_{i} \cong \mathrm{PSL}_{2}(7)$ then $|G: M|$ is a power of 2 , if $L_{i} \cong \mathrm{PSL}_{2}(11)$ then $|G: M|$ is a multiple of either 2 or 3 , and if $L_{i} \cong \mathrm{PSL}_{5}(2)$ then $|G: M|$ is a multiple of either 2 , or 3 , or 7 . Moreover, $|L|_{2}=m_{2}^{n}$ and $|L|_{3}=m_{3}^{n}$.

Proof. Put

$$
p= \begin{cases}7 & \text { if } L_{i} \cong \operatorname{PSL}_{2}(7) \\ 11 & \text { if } L_{i} \cong \operatorname{PSL}_{2}(11) \\ 31 & \text { if } L_{i} \cong \operatorname{PSL}_{5}(2)\end{cases}
$$

The normalizer of a Sylow $p$-subgroup in $L_{i}$ is a maximal subgroup of $L_{i}$ (see, for example, the table above). For $i=1, \ldots, n$, we fix a subgroup $P_{i} \in \operatorname{Syl}_{p}\left(L_{i}\right)$ and put

$$
B_{i}=N_{L_{i}}\left(P_{i}\right) \text { and } P=\left\langle P_{1}, \ldots, P_{n}\right\rangle \cong P_{1} \times \cdots \times P_{n} .
$$

It is clear that $P \in \operatorname{Syl}_{p}(L)$ and

$$
N_{L}(P)=\left\langle B_{1}, \ldots, B_{n}\right\rangle \cong B_{1} \times \cdots \times B_{n} .
$$

By the table above, we have $\left|L_{i}: B_{i}\right|=m, i=1, \ldots, n$. We obtain

$$
\left|L: N_{L}(P)\right|=\left|L_{1}: B_{1}\right| \cdot \ldots \cdot\left|L_{n}: B_{n}\right|=m^{n} .
$$

Using the Frattini argument (see [1, Proposition 6.3]), we conclude that $G=L N_{G}(P)$; hence, we have

$$
\left|G: N_{G}(P)\right|=\left|L: N_{L}(P)\right|=m^{n} \text {. }
$$

Let $M$ be a maximal subgroup of $G$ containing $N_{G}(P)$. Then $|G: M|$ divides $\left|G: N_{G}(P)\right|$ and the equalities $G=L N_{G}(P)=L M$ hold.

\section{ON 3-FRATTINI EXTENSIONS OF PSL 2 (7)}

In this section, we prove Proposition 2.

The following properties of the Frattini subgroup are well known, see [6, Ch. A, Theorems 9.2, 9.3, and 9.7].

Lemma 9. Let $G$ be a finite group.

(1) If $N \unlhd G$ then $\Phi(G) N / N \leq \Phi(G / N)$.

(2) If $N \unlhd G$ and $N \leq \Phi(G)$ then $\Phi(G) / N=\Phi(G / N)$.

(3) If $G$ is a p-group, where $p$ is prime, then $G / \Phi(G)$ is an elementary Abelian p-group; moreover, the least possible number of generators of $G$ is equal to the dimension of $G / \Phi(G)$ (regarded as a vector space over $\mathbb{F}_{p}$ ).

(4) The subgroup $\Phi(G)$ is nilpotent.

Proof. 
The following notion was introduced by Gaschütz [7]. A finite group $F$ is called a $p$-Frattini extension of a group $G$ whose kernel is $N$ if $G \cong F / N$ for a suitable normal $p$-subgroup $N \unlhd F$ such that $N \leq \Phi(F)$. If $N$ is an elementary Abelian group then $F$ is called a p-elementary Frattini extension of $G$.

The following assertion is immediate from Lemma 9.

Lemma 10. If $F$ is a p-Frattini extension of a finite group $G$ whose kernel is $N$ then $F / \Phi(N)$ is a p-elementary Frattini extension of $G$ whose kernel is $N / \Phi(N)$.

Lemma 11. Let a prime number $p$ divide the order of a finite group $G$. Let $\mathscr{E}$ denote the class of all p-elementary Frattini extensions $F$ of $G$ whose kernel is non-trivial. Then the following assertions hold.

(1) We have $\mathscr{E} \neq \varnothing$.

(2) There exists $E_{0} \in \mathscr{E}$ such that, for every $H \in \mathscr{E}$, there exists a homomorphism from $E_{0}$ onto $H$; moreover, the kernel of this homomorphism is contained in the kernel of the extension $E_{0}$.

(3) There exists a set of generators of the kernel of the extension $E_{0}$ consisting of at most $(|G|-1)^{2}$ elements.

Proof. For assertion (1), see [6, Ch. B, Theorem 11.8 or Appendix $\beta$, Corollary $\beta 8$ ]. For assertion (2), see [7;6, Proposition $\beta 5$ ]. Assertion ( 3 ) is a consequence of [6, Propositions $\beta 3$ and $\beta 5$ ].

The group $E_{0}$ from Lemma 11 is called the universal p-elementary Frattini extension of $G$ and its kernel (regarded as an $\mathbb{F}_{p} G$-module) is called the maximal $p$-Frattini module of $G$. It follows from Lemmas 10 and 11 that many properties of $p$-Frattini extensions are consequences of properties of $p$ elementary Frattini extensions and, in particular, of the universal $p$-elementary Frattini extension.

Every group in $\mathscr{U}$ is a 3 -Frattini extension of the group $\mathrm{PSL}_{2}(7)$. We will need more information on 3 -elementary Frattini extensions of $\mathrm{PSL}_{2}(7)$. The following assertion provides us with an example of such an extension whose kernel is non-trivial.

Lemma 12. There exists a 3-elementary Frattini extension of $\mathrm{PSL}_{2}(7)$ such that the order of the kernel is $3^{7}$.

Proof. According to [2], the group $\mathrm{PSL}_{2}(7)$ admits an absolutely irreducible $\mathbb{F}_{3}$-representation of degree 7 such that the second cohomology group is non-trivial.

The following assertion shows that there is no other 3 -elementary Frattini extension of $\mathrm{PSL}_{2}(7)$ whose kernel is non-trivial.

Lemma 13. The maximal 3-Frattini module of the group $\mathrm{PSL}_{2}(7)$ is irreducible and its dimension is 7 .

Proof. See [15, Theorem 1].

As is mentioned in the introduction, in this section, we do not assume that all groups under consideration are finite. Recall that a group $G$ is said to be periodic if the order of each element of $G$ is finite. A $p$-group is a periodic group such that the orders of elements are powers of a fixed prime $p$. A group is said to be locally finite if every finitely generated subgroup of this group is finite. A group $G$ is said to be finitely approximated if, for every element $g \in G$ with $g \neq 1$, there exists an epimorphism $\tau$ from $G$ onto a suitable finite group such that $g^{\tau} \neq 1$.

Recall that a variety of groups is a class of groups axiomatized by a set of identities. The variety generated by a group $G$ is the least variety containing $G$. If an identity holds in $G$ then it holds in every group in the variety generated by $G$. In particular, every group in the variety generated by a group of exponent $m$ is a periodic group of period $m$. Necessary information on varieties of groups can be found in $[13,20]$.

Lemma 14. The following assertions hold.

(1) Let $G$ be a finite group and let $\mathscr{B}$ be the variety generated by $G$. Then the free group of rank $r$ in the variety $\mathscr{B}$ is finite and its order is at most $|G|^{|G|^{r}}$.

(2) The variety generated by a finite group consists of locally finite groups.

(3) The variety generated by a finite p-group consists of locally finite p-groups. 
Proof. For assertion (1), see [20, Theorem 15.71]. Assertion (2) is immediate from assertion (1). Every group in the variety generated by a group of period $p^{m}$ satisfies the identity $x^{p^{m}}=1$. Hence, assertion (3) is a consequence of assertion (2).

Notice that assertions (2) and (3) of Lemma 14, as well as the fact that the free group of a finite rank in the variety generated by a finite group is finite, are consequences of the positive solution of the restricted Burnside problem [12, 24, 25].

Lemma 15. Let a prime number $p$ divide the order of a finite group $G$ and let $r$ be the dimension of the maximal p-Frattini module of $G$. Then, for every variety $\mathscr{B}$ of locally finite $p$-groups, there exists a p-Frattini extension $F$ of $G$ whose kernel is the free group of rank $r$ in the variety $\mathscr{B}$.

Proof. See [4, Assertions 2.1 and 2.2]

We turn to the proof of Proposition 2.

Let $G \in \mathscr{U}$ and let ${ }^{-}: G \rightarrow G / \Phi(G)$ be the natural epimorphism. Consider a maximal subgroup $M$ of $G$. Since $\Phi(G) \leq M$, we find that $\bar{M}$ is a maximal subgroup of $\bar{G} \cong \mathrm{PSL}_{2}(7)$. By Lemma 4 , we find that $\bar{M}$ is a Hall subgroup of $\bar{G}$. By Lemma 6 , we have $3 \in \pi(\bar{M})$. Since $\Phi(G)$ is a 3 -group, we have $\pi(M)=\pi(\bar{M})$. Since $|G: M|=|\bar{G}: \bar{M}|$, we conclude that the order and the index of $M$ are relatively prime, i.e., $M$ is a Hall subgroup. We obtain $G \in \mathscr{V}$, which finishes the proof of assertion (1) of Proposition 2.

By Lemma 3, there exists a subgroup $\bar{H}$ of $\bar{G}$ such that $\bar{G}=\overline{M H}$ and $\bar{M} \cap \bar{H}=1$. Since $\bar{M}$ is a Hall subgroup, the order of $\bar{H}$ is not a multiple of 3. Let $H$ denote the preimage of $\bar{H}$ in $G$. Then $H$ is the extension of the 3 -group $\Phi(G)$ by the $3^{\prime}$-group $\bar{H}$. By the Schur-Zassenhaus Theorem [23, Ch. IV, Theorem 27], there exists a subgroup $N$ of $H$ such that $H=N \Phi(G)$ and $N \cong \bar{H}$. It is easy to see that $N$ is the complement of $M$ in $G$. This finishes the proof of assertion (2) of Proposition 2.

Assertions (3) and (4) of Proposition 2 are immediate from Lemmas 9, 11, 12, and 13.

Assertion (5) of Proposition 2 is a consequence of Lemmas 13 and 15. Let $\mathscr{B}$ denote the variety generated by a finite 7 -generated 3 -group $P$. Then $\mathscr{B}$ consists of locally finite 3 -groups and $P$ is a homomorphic image of the free group of rank 7 in $\mathscr{B}$, see Lemma 14. Hence, assertion (5) implies assertion (6). In particular, for every $m$, the cyclic group of order $3^{m}$ is a homomorphic image of $\Phi(G)$ for a suitable $G \in \mathscr{U}$. Therefore, the exponent of the Frattini subgroup of a group in $\mathscr{U}$ can be arbitrarily large. This finishes the proof of assertion (7) of Proposition 2.

The rest of the section is devoted to the proof of assertion (8) of Proposition 2.

Recall the well-known positive solution of the Burnside problem for solvable groups.

Lemma 16. Every solvable periodic group is locally finite.

Proof. See [5, Proposition 1.1.5].

We will need some properties of the Golod group, which was used in the negative solution of the "unrestricted" Burnside problem [8, 9].

Lemma 17. If $d \geq 2$ and $p$ is a prime number then there exists an infinite finitely approximated $d$-generated p-group.

Proof. See [8, Example 2; 13, Example 18.3.2].

Lemma 18. If $d \geq 2$ and $p$ is a prime number then there exists a sequence $G_{i}, i=1,2, \ldots$, of finite r-generated p-groups such that the degrees of solvability of the groups $G_{i}$ form an unbounded sequence.

Proof. Let $G$ be an infinite finitely approximated $d$-generated $p$-group, see Lemma 17 . By Lemma 16, the group $G$ is unsolvable. For every natural $i$, the group $G^{(i)}$ (the $i$ th derived subgroup of $G$ ) contains a non-trivial element $g_{i}$. Since $G$ is finitely approximated, there exists an epimophism

$$
\tau_{i}: G \rightarrow G_{i}
$$

from $G$ onto a finite group $G_{i}$ with $g_{i}^{\tau_{i}} \neq 1$. Since $g_{i}^{\tau_{i}} \in G_{i}^{(i)}$, the degree of solvability of $G_{i}$ is greater than $i$. It remains to notice that $G_{i}$ is a $d$-generated finite $p$-group. 
We turn to the proof of assertion (8) of Proposition 2.

By Lemma 18, the degrees of solvability of suitable 7 -generated finite 3 -groups $G_{i}, i=1,2, \ldots$, form an unbounded sequence. Each of these groups is a homomorphic image of the Frattini subgroup of a suitable group in $\mathscr{U}$, see assertion (6). Hence, for every natural $n$, there exist a group $G \in \mathscr{U}$ such that the degree of solvability of the group $\Phi(G)$ is greater than $n$. This finishes the proof of the proposition.

\section{STRUCTURE OF A FINITE GROUP WHOSE MAXIMAL SUBGROUPS HAVE THE HALL PROPERTY}

In this section, we prove Theorems 1 and 2 .

Lemma 19. Assume that $G \in \mathscr{V}$, the solvable radical of $G$ is trivial, and $L$ is a minimal normal subgroup of $G$. Then the quotient group $G / L$ is solvable.

Proof. Let $-: G \rightarrow G / L$ be the natural epimorphism. By [1, Propositions 8.2, 8.3], we have $L=$ $L_{1} \times \cdots \times L_{n}$, where non-Abelian simple groups $L_{i}$ are pairwise isomorphic. $>$ From Lemma 4 it follows that each $L_{i}$ is isomorphic to either $\operatorname{PSL}_{2}(7)$, or $\operatorname{PSL}_{2}(11)$, or $\operatorname{PSL}_{5}(2)$. We prove that $\bar{G}$ is a solvable group.

Assume the contrary. As in Lemma 8, put

$$
m= \begin{cases}2^{3} & \text { if } L_{i} \cong \operatorname{PSL}_{2}(7), \\ 2^{2} \cdot 3 & \text { if } L_{i} \cong \operatorname{PSL}_{2}(11), \\ 2^{10} \cdot 3^{2} \cdot 7 & \text { if } L_{i} \cong \operatorname{PSL}_{5}(2) .\end{cases}
$$

Since $\left|L_{i}\right|_{2}=m_{2}$ and $\left|L_{i}\right|_{3}=m_{3}$, we have $|L|_{2}=m_{2}^{n}$ and $|L|_{3}=m_{3}^{n}$. By Lemma 8 , there exists a subgroup $M$ of $G$ such that $G=L M$ and $|G: M|$ divides $m^{n}$. We show that neither 2 nor 3 divides $|G: M|$.

Since $\bar{G}$ is unsolvable, from Lemmas 2 and 4 it follows that some composition factor of $\bar{G}$ is isomorphic to either $\mathrm{PSL}_{2}(7)$, or $\mathrm{PSL}_{2}(11)$, or $\mathrm{PSL}_{5}(2)$. Since 2 and 3 divide the orders of these groups, we have $|\bar{G}|_{2}>1$ and $|\bar{G}|_{3}>1$. We conclude that

$$
\begin{aligned}
& |G|_{2}>|L|_{2}=m_{2}^{n}, \\
& |G|_{3}>|L|_{3}=m_{3}^{n} .
\end{aligned}
$$

If either 2 or 3 divides $|G: M|$ then one of the equations

$$
\begin{aligned}
|G|_{2} & =|G: M|_{2}=m_{2}^{n}, \\
|G|_{3} & =|G: M|_{3}=m_{3}^{n}
\end{aligned}
$$

holds because $|G: M|$ divides $m^{n}$ and $M$ is a Hall subgroup. This contradicts (43).

Since $\pi(m) \subseteq\{2,3,7\}$ and $|G: M|$ divides $m^{n}$, we find that $|G: M|$ is a power of 7 . In particular, it follows from the definition of $m$ that each group $L_{i}$ is isomorphic to $\mathrm{PSL}_{5}(2)$ and $|G: M|=m_{7}^{n}$. Since $M$ is a Hall subgroup of $G$, we have

$$
|G|_{7}=m_{7}^{n}
$$

By the definition of $m$, we conclude that $\left|L_{i}\right|_{7}=m_{7}$ and $|L|_{7}=m_{7}^{n}$.

We prove that each composition factor of $\bar{G}$ is isomorphic to neither $\mathrm{PSL}_{2}(7)$ nor $\mathrm{PSL}_{2}(11)$. Assume the contrary. Then there exists a homomorphic image $\widetilde{G}$ of $\bar{G}$ and a minimal normal subgroup of $\widetilde{G}$ of the form $S_{1} \times \cdots \times S_{k}$, where the groups $S_{i}$ are pairwise isomorphic and each of them is isomorphic to either $\mathrm{PSL}_{2}(7)$ or $\mathrm{PSL}_{2}(11)$. Moreover, the group $\widetilde{G}$ is a homomorphic image of the group $G$ and the subgroup $L$ is contained in the kernel of the corresponding homomorphism. By Lemma 8, there exists a maximal subgroup of $\widetilde{G}$ whose index is a multiple of either 2 or 3 . Let $M$ denote the preimage of this subgroup in $G$. Then $M$ is a maximal subgroup of the same index. Since $M$ contains $L$, either 2 or 3 divides $|M|$. Since $M$ is a Hall subgroup, we arrive at a contradiction.

By Lemma 4, each non-Abelian composition factor of $\bar{G}$ is isomorphic to $\operatorname{PSL}_{5}(2)$. Since $\bar{G}$ is unsolvable, we find that 7 divides $|\bar{G}|=|G: L|$. We obtain $|G|_{7}>|L|_{7}=m_{7}^{n}$, which contradicts (4.3). 
Lemma 20. Assume that $G \in \mathscr{V}$, the solvable radical of $G$ is trivial, and $L$ is a minimal normal subgroup of $G$. Then $L$ is a Hall subgroup of $G$. Moreover, there exists a Hall subgroup $H$ of $G$ such that $G=L H$ and $L \cap H=1$.

Proof. By the Schur-Zassenhaus Theorem [23, Ch. IV, Theorem 27], it suffices to show that the order of $L$ and the index of $L$ in $G$ are relatively prime. Assume that a prime number $p$ divides both $|L|$ and $|G: L|$. Let $\bar{G}=G / L$. By Lemma 19 , the group $\bar{G}$ is solvable. By Lemma 2, each maximal subgroup of $\bar{G}$ is a Hall subgroup.

By the Hall Theorem [10], there exists a $p^{\prime}$-Hall subgroup $\bar{K}$ of $\bar{G}$. Since each maximal subgroup of $\bar{G}$ is a Hall subgroup and the index $|\bar{G}: \bar{K}|$ is a power of $p$, the subgroup $\bar{K}$ coincides with the maximal subgroup containing $\bar{K}$. Let $K$ be the preimage of $\bar{K}$ in $G$. Then $K$ is a maximal subgroup of $G$ (hence, $K$ is a Hall subgroup) and $|G: K|=|\bar{G}: \bar{K}|$ is a power of $p$. Since $K$ contains $L$, we find that $p$ divides $|K|$. Since $K$ is a Hall subgroup, we arrive at a contradiction.

Lemma 21. Let $G \in \mathscr{V}$ and let $G$ be a non-trivial group whose solvable radical is trivial. Then $G$ is isomorphic to either $\mathrm{PSL}_{2}(7)$, or $\mathrm{PSL}_{2}(11)$, or $\mathrm{PSL}_{5}(2)$.

Proof. Let $L$ be a minimal normal subgroup of $G$. By Lemmas 19 and 20 , we have $L=L_{1} \times \cdots \times L_{n}$, where the subgroups $L_{i}$ are conjugated in $G$ and each of them is isomorphic to either $\operatorname{PSL}_{2}(7)$, or $\mathrm{PSL}_{2}(11)$, or $\mathrm{PSL}_{5}(2)$; moreover, there exists a Hall subgroup $H$ of $G$ such that $G=L H, L \cap H=1$, and the action of $H$ on $\left\{L_{i} \mid 1 \leq i \leq n\right\}$ by conjugation is transitive. Hence, $n=\left|H: N_{H}\left(L_{1}\right)\right|$.

It suffices to show that $H=1$. Assume the contrary. Let $\pi=\pi\left(L_{1}\right)$. Consider the quotient group $Q=N_{H}\left(L_{1}\right) / C_{H}\left(L_{1}\right)$. There exists an embedding of $Q$ into Aut $\left(L_{1}\right)$. By Lemma $5, Q$ is a $\pi$-group. On the other hand, $Q$ is a quotient group of a suitable subgroup of $H$. Hence, $Q$ is a $\pi^{\prime}$-group. We conclude that $Q=1$ and $N_{H}\left(L_{1}\right)=C_{H}\left(L_{1}\right)$.

Let $h_{1}, \ldots, h_{n}$ be pairwise distinct representatives of all (right) cosets of $H$ modulo $N_{H}\left(L_{1}\right)$. Then the subgroups $L_{1}^{h_{1}}, \ldots, L_{1}^{h_{n}}$ are pairwise distinct. Without loss of generality, we may assume that $L_{i}=L_{1}^{h_{i}}$. Consider the subset

$$
P=\left\{x^{h_{1}} x^{h_{2}} \ldots x^{h_{n}} \mid x \in L_{1}\right\}
$$

of $G$. It is easy to see that $P$ is a subgroup and $P \cong L_{1}$. We fix an arbitrary element $h \in H$ and prove that $h \in C_{G}(P)$.

Indeed, there exists a permutation $\sigma$ on $\{1, \ldots, n\}$ such that

$$
N_{H}\left(L_{1}\right) h_{i} h N_{H}\left(L_{1}\right) h_{i \sigma} \text {. }
$$

Let $g \in P$. We have $g=x^{h_{1}} x^{h_{2}} \ldots x^{h_{n}}$ for some $x \in L_{1}$ and

$$
g^{h}=\left(x^{h_{1}} x^{h_{2}} \ldots x^{h_{n}}\right)^{h}=x^{h_{1} h} x^{h_{2} h} \ldots x^{h_{n} h} .
$$

Notice that $h_{i} h=c_{i} h_{i \sigma}$, where $c_{i} \in N_{H}\left(L_{1}\right)=C_{H}\left(L_{1}\right)$. If $i \neq j$ then $\left[L_{i}, L_{j}\right]=1$. Hence, we have

$$
g^{h} x^{c_{1} h_{1 \sigma}} x^{c_{2} h_{2 \sigma}} \ldots x^{c_{n} h_{n \sigma}} x^{h_{1 \sigma}} x^{h_{2 \sigma}} \ldots x^{h_{n \sigma}}=x^{h_{1}} x^{h_{2}} \ldots x^{h_{n}}=g .
$$

We obtain $H \leq C_{G}(P)$. In particular, $T=P H$ is a subgroup of $G$. Since $P$ is a $\pi$-group and $H$ is a $\pi^{\prime}$-group, their intersection $P \cap H$ is a trivial group. We find that $|T|=|P||H|$. Let $M$ be a maximal subgroup of $G$ containing $T$. Then $|G: M| \in \pi$ and every number in $\pi$ divides the order of $M$. Since each maximal subgroup of $G$ is a Hall subgroup, we arrive at a contradiction. Thus, $H=1$.

Lemma 22. Let $G \in \mathscr{V}$ be an unsolvable group and let $\widetilde{G}=G / S(G)$. Then one of the following assertions holds.

(1) The group $\widetilde{G}$ is isomorphic to either $\mathrm{PSL}_{2}(7)$, or $\mathrm{PSL}_{2}(11)$, or $\mathrm{PSL}_{5}(2), S(G)$ is a Hall subgroup of $G$, and $G$ is a splittable extension of $S(G)$ by $\widetilde{G}$.

(2) We have $\widetilde{G} \cong \mathrm{PSL}_{2}(7)$ and $(|S(G)|,|G: S(G)|)=3$. 
Proof. Assume that $(|S(G)|,|G: S(G)|) \neq 1$. Then there exists a prime $p$ dividing the orders of $S(G)$ and $\widetilde{G}$.

Assume that $p$ divides the index of a maximal subgroup $\widetilde{M}$ of $\widetilde{G}$. Let $M$ be the preimage of $\widetilde{M}$ in $\widetilde{G}$. Since $M$ is a maximal subgroup, it is a Hall subgroup. Since $S(G) \leq M$, we find that $p$ divides both $|M|$ and $|G: M|=|\widetilde{G}: \widetilde{M}|$. We arrive at a contradiction. Hence, $p$ divides the index of no maximal subgroup of $\widetilde{G}$.

By Lemma 2, we have $\widetilde{G} \in \mathscr{V}$. Since $S(\widetilde{G})=1$, from Lemma 21 it follows that $\widetilde{G}$ is isomorphic to either $\mathrm{PSL}_{2}(7)$, or $\mathrm{PSL}_{2}(11)$, or $\mathrm{PSL}_{5}(2)$. $>$ From Lemma 7 it follows that $\widetilde{G} \cong \mathrm{PSL}_{2}(7)$ and $(|S(G)|,|G: S(G)|)$ is a power of 3 . Since $\left|\mathrm{PSL}_{2}(7)\right|_{3}=3$, assertion (2) of the lemma holds.

If $(|S(G)|,|G: S(G)|)=1$ then, by the Schur-Zassenhaus Theorem [23, Ch. IV, Theorem 27], there exists a complement of $S(G)$ in $G$. It remains to use Lemma 21 .

Lemma 23. Assume that $G \in \mathscr{V}$ and $S=S(G)$. Let $P$ be a non-trivial normal $p$-subgroup of $G$, where $p$ is prime, and let ${ }^{\sim}: G \rightarrow G / P$ be the natural epimorphism. Then either $\widetilde{S}$ is a $p^{\prime}-$ group or the $p^{\prime}$-Hall subgroup of $S$ is a normal subgroup of $G$.

Proof. Notice that $P \leq S$. Assume that $\widetilde{S}$ is not a $p^{\prime}$-group, i.e., let $p$ divide $|\widetilde{S}|$. If $\widetilde{H} \in \operatorname{Hall}_{p^{\prime}}(\widetilde{S})$ then $\widetilde{H}<\widetilde{S}$. We prove that $\widetilde{H} \unlhd \widetilde{S}$.

Assume the contrary. Then $\widetilde{S} \not \leq N_{\widetilde{G}}(\widetilde{H})$. By the Hall Theorem [10], all $p^{\prime}$-Hall subgroups of $\widetilde{S}$ are pairwise conjugated in $\widetilde{S}$. By the Frattini argument [1, Proposition 6.3], we have $N_{\widetilde{G}}(\widetilde{H}) \widetilde{S}=\widetilde{G}$. If $\widetilde{M}$ is

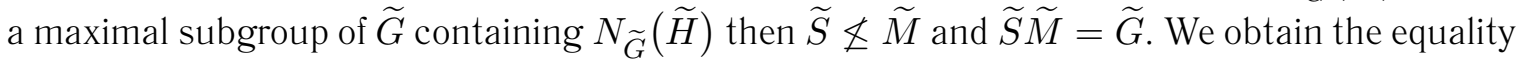

$$
|\widetilde{G}: \widetilde{M}|=|\widetilde{S}: \widetilde{S} \cap \widetilde{M}| \text {. }
$$

Since $\widetilde{H} \leq \widetilde{S} \cap \widetilde{M}$, the index $|\widetilde{G}: \widetilde{M}|$ is a non-trivial power of $p$. Let $M$ be the preimage of $\widetilde{M}$ in $G$. Then $p$ divides $|G: M|=|\widetilde{G}: \widetilde{M}|$ and $|M|=|\widetilde{M}||P|$. Since $M$ is a Hall subgroup, we arrive at a contradiction,

Thus, we have $\widetilde{H} \unlhd \widetilde{S}$ and $\widetilde{H}=O_{p^{\prime}}(\widetilde{S})$. Let $H$ be the preimage of $\widetilde{H}$ in $G$. Then $H \unlhd S$. By the SchurZassenhaus Theorem [23, Ch. IV, Theorem 27], there exists a complement $V \cong \widetilde{H}$ of $P$ in $H$. Notice that $V \in \operatorname{Hall}_{p^{\prime}}(H) \subseteq \operatorname{Hall}_{p^{\prime}}(S)$.

Assume that $V$ is not a normal subgroup of $G$ and $M$ is a maximal subgroup containing $N_{G}(V)$.

The following equations hold:

$$
\begin{aligned}
& N_{S}(V) P=N_{S}(V) V P=N_{S}(V) H, \\
& N_{G}(V) P=N_{G}(V) V P=N_{G}(V) H .
\end{aligned}
$$

By the Hall Theorem [10], all $p^{\prime}$-Hall subgroups of the solvable group $H$ are conjugated in $H$. By the Frattini argument [1, Proposition 6.3], we obtain $N_{S}(V) H=S$. Therefore, $N_{S}(V) P=S$. Similar arguments show that $N_{G}(V) H=G$.

We have $|S: P|=\left|N_{S}(V): N_{S}(V) \cap P\right|$. By assumption, $p$ divides $|\widetilde{S}|=|S: P|$. Hence, $p$ divides $\left|N_{S}(V)\right|$ and, consequently, it divides both $\left|N_{G}(V)\right|$ and $|M|$.

Since $N_{G}(V) H=G$, we obtain $M H=G$. Hence, $|G: M|=|H: M \cap H|$. Since $V \leq M \cap H$, we find that $|H: M \cap H|$ divides $|H: V|=|P|$. Hence, $|G: M|$ is a non-trivial power of $p$. Since $M$ is a Hall subgroup of $G$, we arrive at a contradiction.

Lemma 24. If $G \in \mathscr{V}, G / S(G) \cong \mathrm{PSL}_{2}(7)$, and $H \in \operatorname{Hall}_{3^{\prime}}(S(G))$ then $H \unlhd G$.

Proof. Assume the contrary. Then there exists a proper maximal subgroup $M$ of $G$ containing $N=N_{G}(H)$. By the Hall Theorem [10], all $3^{\prime}$-Hall subgroups are pairwise conjugated in $S=S(G)$. By the Frattini argument [1, Proposition 6.3], we have $N S=G$. By the Homomorphism Theorem, we obtain

$$
\operatorname{PSL}_{2}(7) \cong G / S \cong N /(N \cap S)
$$


We find that 3 divides both $|N|$ and $|M|$. On the other hand, $|G: M|$ divides $|G: N|=|S:(S \cap N)|$. Since $H \leq S \cap N$, we conclude that $|S:(S \cap N)|$ is a power of 3 . Hence, the index $|G: M|$ is a power of 3 . Since $M$ is a Hall subgroup of $G$, we arrive at a contradiction.

Lemma 25. Let $G \in \mathscr{V}$. Then $G$ admits a normal series

$$
G=G_{0}>G_{1}>\cdots>G_{n}=1
$$

such that

(1) if $i>1$ then $G_{i-1} / G_{i}$ is an elementary Abelian $p_{i}$-group for a suitable $p_{i} \in \pi(G)$, this group is isomorphic to a Sylow $p_{i}$-subgroup of $G$, and the action of $G / G_{i}$ on $G_{i-1} / G_{i}$ is irreducible;

(2) the group $G_{1}$ is a Hall subgroup of $G$ and either $\bar{G}=G / G_{1}$ is a cyclic group of prime order, or $\bar{G}$ is isomorphic to one of the simple groups $\mathrm{PSL}_{2}(11)$, $\mathrm{PSL}_{5}(2)$, or we have $\bar{G} \in \mathscr{U}$.

Proof. If $G$ is a solvable group then the required assertion is immediate from Proposition 1.

Assume that $G$ is unsolvable. Let $G_{1}$ be a $3^{\prime}$-Hall subgroup of $S(G)$. We show that $G_{1} \unlhd G$ and assertion (2) of the lemma holds for the quotient group $\bar{G}=G / G_{1}$.

By Lemmas 2 and 21, the quotient group $G / S(G)$ is isomorphic to either $\mathrm{PSL}_{2}(7)$, or $\mathrm{PSL}_{2}(11)$, or $\mathrm{PSL}_{5}(2)$. In particular, the index of $S(G)$ is a multiple of 3. By Lemma 22, either (a) $S(G)$ is a Hall subgroup of $G$ or (b) we have $G \cong \mathrm{PSL}_{2}(7)$ and $(|S(G)|,|G: S(G)|)=3$.

If (p) holds then $S(G)$ is a $3^{\prime}$-subgroup; hence, $G_{1}=S(G) \unlhd G$. It is clear that assertion (2) holds.

Assume that (b) holds, i.e., let $G \cong \mathrm{PSL}_{2}(7)$ and $(|S(G)|,|G: S(G)|)=3$. Then the $3^{\prime}$-Hall subgroup $G_{1}$ of $S(G)$ is a normal subgroup of $G$ in view of Lemma 24 . Each maximal subgroup of $\bar{G}=G / G_{1}$ is a Hall subgroup in view of Lemma 2. > From the definition of $G_{1}$ it follows that $S(\bar{G})=S(G) / G_{1}$ is a 3 -group. We have $\bar{G} / S(\bar{G}) \cong \mathrm{PSL}_{2}(7)$. We prove that $\Phi(\bar{G})=S(\bar{G})$, which, in particular, implies that $\Phi(\bar{G})$ is a 3 -group.

By Lemma 9, the Frattini subgroup is nilpotent; hence, $\Phi(\bar{G}) \leq S(\bar{G})$.

We show that $S(\bar{G}) \leq \Phi(\bar{G})$. Let $\bar{M}$ be a maximal subgroup of $\bar{G}$. If $S(\bar{G}) \not \leq \bar{M}$ then $\bar{G}=\bar{M} S(\bar{G})$. We conclude that the index $|\bar{G}: \bar{M}|$ is a power of 3 . Since

$$
\bar{M} /(\bar{M} \cap S(\bar{G})) \cong \bar{G} / S(\bar{G}) \cong \mathrm{PSL}_{2}(7),
$$

the order $|\bar{M}|$ is a multiple of 3 . By Lemma 2 , we have $\bar{G} \in \mathscr{V}$. We conclude that $\bar{M}$ is a Hall subgroup of $\bar{G}$, a contradiction. Therefore, $S(\bar{G})$ is contained in every maximal subgroup of $\bar{G}$; hence, it is contained in the intersection $\Phi(\bar{G})$ of all such subgroups.

Thus, if $G$ is an unsolvable group whose maximal subgroups have the Hall property then $G$ admits a normal series

$$
G=G_{0}>G_{1} \geq 1
$$

such that either the quotient group $\bar{G}=G_{0} / G_{1}$ is isomorphic to a simple group in $\left\{\mathrm{PSL}_{2}(11), \mathrm{PSL}_{5}(2)\right\}$ or $\Phi(\bar{G})$ is a 3 -group and $\bar{G} / \Phi(\bar{G}) \cong \mathrm{PSL}_{2}(7)$. Moreover, $G_{1}$ is a Hall subgroup of $G_{0}=G$.

Using induction on $n=\left|\pi\left(G_{1}\right)\right|$, we prove that series (4.4) can be extended to the required normal series

$$
G=G_{0}>G_{1}>\cdots>G_{n}=1
$$

of $G$. For $\left|\pi\left(G_{1}\right)\right|=0$, this assertion is already proven.

We prove this assertion for $\left|\pi\left(G_{1}\right)\right|=1$. In this case, $G_{1}$ is a $p$-group, where $\pi\left(G_{1}\right)=\{p\}$. Assume that $P \leq G_{1}, P \neq G_{1}$, and $P$ is a minimal normal subgroup of $G$. Consider the quotient group $\widetilde{G}=G / P$. By the Schur-Zassenhaus Theorem [23, Ch. IV, Theorem 27], we have $\widetilde{G}=\widetilde{Q} \widetilde{G}_{1}$, where $\widetilde{G}_{1}$ is the image of the $p$-subgroup $G_{1}$ in $\widetilde{G}$ and $\widetilde{Q}$ is a proper subgroup of $\widetilde{G}$ that is isomorphic to $G / G_{1}$. Let $\widetilde{M}$ be 
a maximal subgroup of $\widetilde{G}$ containing $\widetilde{Q}$ and let $M$ be the preimage of $\widetilde{M}$ in $G$. Then $M$ is a maximal subgroup of $G$; hence, this is a Hall subgroup. Since $P \leq M$, the order of $M$ is a multiple of $p$. Hence, $p$ cannot divide $|G: M|=|\widetilde{G}: \widetilde{M}|$. But $|\widetilde{G}: \widetilde{M}|$ divides $|\widetilde{G}: \widetilde{Q}|=\left|\widetilde{G}_{1}\right|$; hence, the former index is a power of $p$. We arrive at a contradiction. Since $P$ is a minimal normal subgroup, we find that $G_{1}=P$ is an elementary Abelian group and the action of $G$ on $G_{1}$ (regarded as a vector space) is irreducible. The series

$$
G=G_{0}>G_{1}>G_{2}=1
$$

satisfies the conclusion of the lemma. Thus, the lemma is valid for $\left|\pi\left(G_{1}\right)\right|=1$.

Assume that the conclusion of the lemma fails for some $n=\left|\pi\left(G_{1}\right)\right|$. Choose the least such $n$.

Let $A$ be a minimal normal subgroup of $G$ that is contained in $G_{1}$. Then $A$ is an elementary Abelian $p$-group for some $p \notin \pi\left(G_{0} / G_{1}\right)$. By Lemma 23 , either $S(G) / A$ is a $p^{\prime}$-group or the $p^{\prime}$-Hall subgroup of $S(G)$ is a normal subgroup of $G$.

Assume that $S(G) / A$ is a $p^{\prime}$-group. Consider the quotient group $\widetilde{G}=G / A$. Since $\left|\pi\left(\widetilde{G}_{1}\right)\right|=n-1$, the group $\widetilde{G}$ admits a normal series

$$
\widetilde{G}=\widetilde{G}_{0}>\widetilde{G}_{1}>\cdots>\widetilde{G}_{n-1}=1
$$

satisfying the conclusion of the lemma. We find that

$$
G=G_{0}>G_{1}>\cdots>G_{n-1}>G_{n}=1,
$$

where $G_{i}$ is the preimage of $\widetilde{G}_{i}, i=0,1, \ldots, n-1$, is a normal series of $G$ satisfying the conclusion of the lemma.

Therefore, the Hall $p^{\prime}$-subgroup $H$ of $S(G)$ is a normal subgroup of $G$. We have $\pi\left(G_{1} / H\right)=\{p\}$ and no minimal normal subgroup of $G^{*}=G / H$, except for $A^{*}=H A / H$, is contained in $G_{1}^{*}=G_{1} / H$. Since $p \notin \pi\left(G / G_{1}\right)$, we have $p \neq 3$. By the above, $A^{*}$ is a Sylow $p$-subgroup of $G^{*}$. Since $A \cong A^{*}$ and $H \in \operatorname{Hall}_{p^{\prime}}(G)$, we find that $G / A$ and $S(G) / A$ are $p^{\prime}$-groups. This case was considered earlier. Thus, in each case, the conclusion of the lemma holds.

Lemma 26. Let a group $G$ admit a normal series

$$
G=G_{0}>G_{1}>\cdots>G_{n}=1
$$

such that, for every $i>1$, the quotient group $G_{i-1} / G_{i}$ is an elementary Abelian $p_{i}$-group for a suitable $p_{i} \in \pi(G)$ and the action of the group $G / G_{i}$ on $G_{i-1} / G_{i}$ (regarded as a vector space) is irreducible. Let $M$ be a maximal subgroup of $G$. Then, for every $i>1$, we have either $G_{i-1} \leq M G_{i}$ or $M \cap G_{i-1}=G_{i}$.

Proof. Assume that $G_{i-1} \not \leq M G_{i}$ for some $i>1$. Since $M$ is a maximal subgroup, from $M G_{i} \neq G$ it follows that $M=M G_{i} \geq G_{i}$ and from $G_{i-1} \not \leq M$ it follows that $G=M G_{i-1}$. Let ${ }^{\sim}: G \rightarrow G / G_{i}$ be the natural epimorphism. The subgroup $\widetilde{M} \cap \widetilde{G}_{i-1}$ of the elementary Abelian group $\widetilde{G}_{i-1}$ is invariant with respect to the action of the group $\widetilde{G}=\widetilde{M} \widetilde{G}_{i-1}$. Since the action of $\widetilde{G}$ on $\widetilde{G}_{i-1}$ is irreducible and $G_{i-1} \not \leq M$, we obtain $\widetilde{M} \cap \widetilde{G}_{i-1}=1$, i.e., $M \cap G_{i-1}=G_{i}$.

Lemma 27. Let a group $G$ admit a normal series

$$
G=G_{0}>G_{1}>\cdots>G_{n}=1
$$

such that the following conditions hold:

(1) for every $i>1$, the quotient group $G_{i-1} / G_{i}$ is an elementary Abelian group that is isomorphic to a suitable Sylow subgroup of $G$ and the action of $G / G_{i}$ on $G_{i-1} / G_{i}$ (regarded as a vector space) is irreducible;

(2) either $\bar{G}=G / G_{1}$ is a cyclic group of prime order, or $\bar{G}=G / G_{1}$ is isomorphic to one of the groups in $\left\{\mathrm{PSL}_{2}(11), \mathrm{PSL}_{5}(2)\right\}$, or $\Phi(\bar{G})$ is a 3 -group with $\bar{G} / \Phi(\bar{G}) \cong \mathrm{PSL}_{2}(7)$.

Then each maximal subgroup of $G$ is a complemented Hall subgroup. 
Proof. Let $M$ be a maximal subgroup of $G$. We prove that $M$ is a Hall subgroup. Consider the least $i \in\{1, \ldots, n\}$ with $G_{i} \leq M$.

Assume that $i>1$. Since $G_{i-1} \not \leq M=M G_{i}$, from Lemma 26 it follows that $M \cap G_{i-1}=G_{i}$. Since $M$ is maximal, we have $G=M G_{i-1}$. We obtain

$$
|G: M|=\left|M G_{i-1}: M\right|=\left|G_{i-1}:\left(M \cap G_{i-1}\right)\right|=\left|G_{i-1}: G_{i}\right|,
$$

i.e., the index of $M$ coincides with the order of a suitable Sylow subgroup $P$ of $G$. We conclude that $M$ is a Hall subgroup and $P$ is its complement.

Let $i=1$, i.e., let $G_{1} \leq M$. Let $\bar{M}$ be the image of $M$ in $\bar{G}=G / G_{1}$. Then $\bar{M}$ is a maximal subgroup of $\bar{G}$. We show that $\bar{M}$ is a Hall subgroup of $\bar{G}$. This is obvious if the order of $\bar{G}$ is prime; otherwise, we apply Lemma 3 and assertion (1) of Proposition 2. Since $G_{1}$ is a Hall subgroup of $G$, the numbers $|G: M|=|\bar{G}: \bar{M}|,|\bar{M}|=\left|M: G_{1}\right|$, and $\left|G_{1}\right|$ are pairwise relatively prime. Hence, the numbers $|G: M|$ and $|M|=|\bar{M}|\left|G_{1}\right|$ are relatively prime too. We conclude that $M$ is a Hall subgroup of $G$.

By Lemma 3 and assertion (2) of Proposition 2, there exists a complement $\bar{H}$ of $\bar{M}$ in $\bar{G}$. Let $H \leq G$ be a subgroup such that $G_{1} \leq H$ and $\bar{H}=H / G_{1}$. By the Schur-Zassenhaus Theorem [23, Ch. IV, Theorem 27], there exists a complement $N$ of $G_{1}$ in $H$. It is easy to see that $N$ is a complement of $M$ in $G$.

The assertion of Theorem 1 is valid in view of Lemmas 25 and 27.

The assertion of Theorem 2 is valid in view of Theorem 1 and Lemma 27.

\section{ACKNOWLEDGMENTS}

The authors are greatful to A. S. Kondrat'ev, V. D. Mazurov, E. I. Khukhro, A. V. Zavarnitsin, and A. V. Vasil'ev for useful discussions.

The work partially supported by the Russian Foundation for Basic Research (grants 10-01-00324, 10-01-00391, and 10-01-90007), the Federal Target Program "Scientific and scientific-pedagogical personnel of innovative Russia" (grant 14.740.11.0346), the Council for President's Grants and Support of Young Russian Scientists (project MK-3395.2012.1), the Program for joint research of SB RAS and UB RAS (project 12-C-1-1018), the grant program of UB RAS for young scientists (project $\mathrm{A}_{3}$ ), and the target program of SB RAS for 2012-2014 (integration project № 14).

\section{REFERENCES}

1. M. Aschbacher, Finite Group Theory (Cambridge Univ. Press, Cambridge, 1986).

2. ATLAS of Finite Group Representations (http://brauer.maths.gmul.ac.uk/Atlas/).

3. J. H. Conway, R. T. Curtis, S. P. Norton, R. A. Parker, and R. A. Wilson, Atlas of Finite Groups. Maximal Subgroups and Ordinary Characters for Simple Groups (Clarendon Press, Oxford, 1985).

4. J. Cossey, O. H. Kegel, and L. G. Kovács, "Maximal Frattini extensions," Arch. Math. 35 (3), 210-217 (1980).

5. M. R. Dixon, Sylow Theory, Formations, and Fitting Classes in Locally Finite Groups (World Scientific, River Edge, NJ, 1994).

6. K. Doerk and T. Hawkes, Finite Soluble Groups (Walter de Gruyter, Berlin-New York, 1992).

7. W. Gaschütz, "Über modulare Darstellungen endlicher Gruppen, die von freien Gruppen induziert werden," Math. Z. 60 3, 274-286 (1954) [in German].

8. E. S. Golod, “On nil-algebras and finitely approximable $p$-groups,” Izv. Akad. Nauk SSSR Ser. Mat. 28 (2), 273-276 (1964) [in Russian].

9. E. S. Golod and I. R. Shafarevich, "On the class field tower," Izv. Akad. Nauk SSSR Ser. Mat. 28 (2), 261272 (1964) [in Russian].

10. P. Hall, “A note on soluble groups,” J. London Math. Soc. 3, 98-105 (1928).

11. P. Hall, “Theorems like Sylow's," Proc. London Math. Soc. (3) 6 (22), 286-304 (1956).

12. P. Hall and G. Higman, "On the $p$-length of $p$-soluble groups and reduction theorem for Burnside's problem," Proc. London Math. Soc. (3) 6 (21), 1-42 (1956). 
13. M. I. Kargapolov and Yu. I. Merzlyakov, Fundamentals of Group Theory (Nauka, Moscow, 1982) [in Russian].

14. A. S. Kondrat'ev, Groups and Lie Algebras (IMM UB RAS, Ekaterinburg, 2010) [in Russian].

15. S. D. Kozlov, "Maximal Frattini $p$-modules of minimal unsolvable groups having cyclic Sylow $p$-subgroups," Algebra and Logic 24 (3), 229-239 (1985) [Algebra i logika 24 (3), 352-364 (1985)].

16. V. M. Levchuk and A. G. Likharev, "Finite simple groups with complemented maximal subgroups," Siberian Math. J. 47 (4), 659-668 (2006) [Sibirsk. Mat. Zh. 47 (4), 798-810 (2006)].

17. N. V. Maslova, "Nonabelian composition factors of a finite group whose all maximal subgroups are Hall," Siberian Math. J. 53 (5), 853-861 (2012) [Sibirsk. Mat. Zh. 53 (5), 1065-1076 (2012)].

18. V. D. Mazurov and E. I. Khukhro (eds.), The Kourovka Notebook (IM SB RAS, Novosibirsk, 2010).

19. V. S. Monakhov, "Finite $\pi$-solvable groups whose maximal subgroups have the Hall property," Math. Notes 84 (3-4), 363-366 (2008) [Mat. Zametki 84 (3), 390-394 (2008)].

20. H. Neumann, Varieties of Groups ( Springer-Verlag, Berlin-Heidelberg-New York, 1967).

21. V. N. Tyutyanov, "Finite groups with complemented subgroups," Izv. F. Skorina Gomel' State Univ. 36 (3), 178-183 (2006) [in Russian].

22. V. N. Tyutyanov and T. V. Tikhonenko, "Finite groups whose maximal subgroups have the Hall property," Izv. F. Skorina Gomel' State Univ. 50 (5), 198-206 (2008) [in Russian].

23. H. Zassenhaus, Lehrbuch der Gruppentheorie, Bd. I (Teubner, Leipzig-Berlin, 1937) [in German].

24. E. I. Zel'manov, "Solution of the restricted Burnside problem for groups of odd exponent," Math. USSR-Izv. 36 (1), 41-60 (1991) [Izv. Akad. Nauk SSSR Ser. Mat. 54 (1), 42-59 (1990)].

25. E. I. Zel'manov, "Solution of the restricted Burnside problem for 2-groups," Math. USSR-Sb. 72 (2), 543565 (1992) [Mat. Sb. 182 (4), 568-592(1991)]. 